\title{
Contagious Obesity
}

\author{
Mario Pappagallo* \\ University La Sapienza of Rome, Italy
}

Submission: July 19, 2018; Published: August 06, 2018

*Corresponding author: Mario Pappagallo, University La Sapienza of Rome, Italy, Email: mariopappagallo3@gmail.com

\section{Opinion}

The social influences, the behavioral norms and the image that everyone has of themselves can determine some morbid conditions such as obesity. It is called "social contagion".Obesity is a potentially fatal disease, reduces the life expectancy of 10 years, causes social unrest and often, among children and adolescents, promotes episodes of bullying, which several times have reported. And yet, the institutions, to date, have looked elsewhere. In fact, obesity is one of the main public health problems in the world both because its prevalence is constantly and worryingly increasing not only in Western countries, but also in those with low-middle income, and because it is an important factor of risk for various chronic diseases, such as type 2 diabetes, cardiovascular disease and cancer.It is estimated that $44 \%$ of cases of type 2 diabetes, $23 \%$ of cases of ischemic heart disease and up to $41 \%$ of some cancers are attributable to obesity / overweight. In total, overweight and obesity represent the fifth most important risk factor for overall mortality and deaths attributable to obesity are at least 2.8 million / year in the world.In Europe, the prevalence of obesity has tripled in many countries since the 1980 s and continues to grow at an alarming rate, especially among children. According to recent WHO estimates, one in two people in Europe is overweight or obese, while one in five is obese. Among the 11-year-olds, one in three is overweight or obese.

Therefore, to clarify whether obesity rates in some contexts depend on a form of social contagion rather than a natural selfselection among similar, possibly also linked to the sharing of places and habits, could change the approaches of health policies for the improvement of the state of health of the populations.In this regard, some Californian researchers - in an article recently published in the journal JAMA Pediatrics - have compared several military families assigned to different territorial locations of unforeseeable residence, therefore without any influence in the choice by the subjects, to study the relationship between the relative rates of local obesity and the development of overweight and obesity in parents and boys.Drawing on the data from an epidemiological study conducted on 38 US military sites, including 1,519 families, of which 1,314 adults and 1,111 adolescents, anthropometric data and body mass index (BMI) were recorded for a year of observation.

As a result, families trafficked in areas with a higher rate of obesity have an average BMI and a higher rate of overweightobesity. For example, for every 1\% more than the rate of territorial obesity we observed: a score of 0.08 more on the BMI figure and a $5 \%$ more obesity on average in adult parents; a $4 \%$ increase in the rate of overweight-obesity in children, children and adolescents.The correlation between the rate of territorial obesity, the BMI values and the prevalence of overweightobesity was stronger for longer periods of settlement $(>24$ months) for boys, and for the whole family it was greater for those who lived outside the settlement rather than inside. "The observation performed on these populations, assigned to territories randomly, would exclude self-selection hypotheses, as well as the analysis on the influence of the possible sharing of certain environments and spaces has also excluded this as a possible confounding factor on the results", comments the Italian Association of Doctors Diabetologists (AMD) on his website where he reports the American pediatric study.

\section{Conclusion}

In conclusion, according to the authors of the research, living in areas with a higher rate of obesity (see Campania, ed) can lead to a greater risk of overweight-obesity. There is, however, a big limitation in the study: that is, that most of the data were self-reported by the observed subjects. So, open road to a new study with selected subjects, analyzed in clinical histories and submitted to ad hoc questionnaires ... So, obesity would be contagious like a virus, a bacterium? Brilliant analogy, which also needs more observations and more consolidated data, but which seems to have a solid basis if we talk about cultural contagion, social contagion.The fact remains that, worldwide, obesity is a potentially fatal disease, reduces the life expectancy of 10 years, causes social unease and often, among children and adolescents, promotes episodes of bullying, which repeatedly chronicles have reported. Yet, the institutions, until today, have 
looked elsewhere. Obesity is one of the main problems of public health both because its prevalence is constantly and worryingly increasing not only in Western countries but also in those with low-middle income and because it is an important risk factor for various chronic diseases, such as type 2 diabetes mellitus, cardiovascular diseases and tumors. But beware, obesity is a disease and it is not the fault of those who suffer from being obese. Assigning to a patient the guilt of his illness is a medical alibi, social justification, a reflection as unjust as ancient (it was the first reaction to the AIDS epidemic, remember? And even before to leprosy, to tuberculosis ... to cancer). So it is not ethical to blame the patient for the therapeutic impotence and even less ethical is to make it stigma. Obesity is the most visible among diseases. The disease can be seen literally before even distinguishing the patient's traits. And the disease is uniquely attributed to a behavior: excessive consumption of food. We are wrong to blame the obese person. Saying "you are fat because you choose to eat a lot" is ethically incorrect and scientifically wrong: the energy balance of a person is at the center of many factors, largely unknown, whose interactions are very complicated. Always to be on the subject, then, who is fat pay. This deresponsibilization of society is well exemplified by the fact that obesity, although considered a disease, does not have a ticket exemption code, so the person must participate in the costs of treatment necessary to prevent and manage obesity. And they are not just new treatments, since the correct approach to obesity requires a multidisciplinary team similar to the one planned for diabetes but even more extensive. Managing obesity well would reduce enormous social and health costs, speaking of $2 \%$ of GDP, caused by its consequences. And what are the responsibilities of society and politics? Many.

For example, leaving citizens-consumers alone against stimulus dealers. Advertising without ethics. Citizens and those who tend to obesity must accept the consequences of an approach that allows food companies to use every trick to convince people to eat more than necessary and to prefer substances with huge amounts of fat, salts and added sugars: substances that activate pleasure centers in a way similar to what happens with drugs. They create addiction. Food addiction. And consequent possible overweight or obesity. Incorrect lifestyles among young people. In 2016, $19.8 \%$ of the population aged 14 and over claims to be a smoker (around 10 million and 400 thousand people), $22.6 \%$ have smoked in the past and $56.1 \%$ have never smoked. The population of 18 years and overweight is $35.9 \%$ (35.5\% overweight, $10.4 \%$ obese), while $51 \%$ are in a normal weighted condition and $3.1 \%$ are underweight $64.7 \%$ of the population aged 11 and over consumed at least one alcoholic beverage during the year: $51.7 \%$ drink wine, $47.8 \%$ consume beer and $43.2 \%$ alcoholic, bitter aperitifs , spirits or liqueurs. 23 million 85 thousand people (39.2\% of the population aged 3 and over) declare that they do not practice sport or physical activity in their free time.Gender differences are high: $43.4 \%$ of women are sedentary compared to $34.8 \%$ of men.

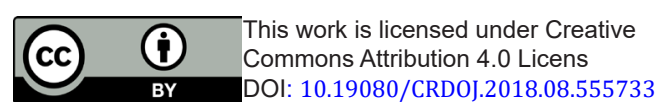

